\title{
Molecular variance analysis (AMOVA) and levels of genetic diversity of complete genome of SARS-CoV- 2 virus from of six South American Countries
}

Pierre Teodosio Felix ( $\square$ pierrefelix@univisa.edu.br)

Laboratory of Population Genetics and Computational Evolutionary Biology - LaBECom, UNIVISA https://orcid.org/0000-0002-9159-7206

\section{Robson da Silva Ramos}

Laboratory of Population Genetics and Computational Evolutionary Biology - LaBECom, UNIVISA https://orcid.org/0000-0002-9935-2633

\section{Sara da Silva Nascimento}

Laboratory of Population Genetics and Computational Evolutionary Biology - LaBECom, UNIVISA https://orcid.org/0000-0002-0090-2456

\section{Willams Felipe Ferreira Silva}

Laboratory of Population Genetics and Computational Evolutionary Biology - LaBECom, UNIVISA https://orcid.org/0000-0001-8210-8442

\section{Maria Dara Pereira da Silva}

Laboratory of Population Genetics and Computational Evolutionary Biology - LaBECom, UNIVISA Bianca Sarah Ferreira dos Santos

Laboratory of Population Genetics and Computational Evolutionary Biology - LaBECom, UNIVISA https://orcid.org/0000-0002-2674-0053

\section{Cícero Batista do Nascimento Filho}

Laboratory of Population Genetics and Computational Evolutionary Biology - LaBECom, UNIVISA https://orcid.org/0000-0002-2356-770X

\section{Antônio João Paulino}

Laboratory of Population Genetics and Computational Evolutionary Biology - LaBECom, UNIVISA https://orcid.org/0000-0003-1870-2695

\section{Dallynne Bárbara Ramos Venâncio}

Laboratory of Population Genetics and Computational Evolutionary Biology - LaBECom, UNIVISA https://orcid.org/0000-0001-7818-1997

\section{Research Article}

Keywords: SARS-CoV-2, COVID-19, Coronavirus, Phylogeny; AMOVA, Bioinformatics, South America 
Posted Date: September 16th, 2020

DOl: https://doi.org/10.21203/rs.3.rs-76869/v1

License: (c) (i) This work is licensed under a Creative Commons Attribution 4.0 International License. Read Full License 


\title{
Molecular variance analysis (AMOVA) and levels of genetic diversity of complete genome of SARS-CoV-2 virus from of six South American Countries
}

Pierre Teodósio Felix*; Robson da Silva Ramos; Sara da Silva Nascimento; Willams Felipe Ferreira Silva; Maria Dara Pereira da Silva; Bianca Sarah Ferreira dos Santos; Cícero Batista do Nascimento Filho; Antônio João Paulino; Dallynne Bárbara Ramos Venâncio

Laboratory of Population Genetics and Computational Evolutionary Biology LaBECom, UNIVISA, Vitória de Santo Antão, Pernambuco, Brazil. *Corresponding author/ Contact: pierrefelix@univisa.edu.br

Keywords: COVID-19, SARS-CoV-2, Coronavirus, Phylogeny; AMOVA, Bioinformatics, South America

\begin{abstract}
Although some Countries in South America have implemented laboratory and patient management protocols for the new coronavirus, the lack of access to basic sanitation and hygiene measures, as well as the lack of drugs and vaccines, has significantly interfered with the epidemiological mechanics of the virus, emphasizing its implications. Therefore, trying to understand the evolutionary aspects of the virus, emerges as another strategy that can help in the most varied measures of prophylaxis. In this work, we evaluated the levels of genetic diversity in 38 complete Genomes of SARS-CoV-2 from six countries in South America, using specific methodologies for paired FST, AMOVA, mismatch, demographic and spatial expansions, molecular diversity and for the time of evolutionary divergence. The analyses showed non-significant evolutionary divergences within and between the six countries, as well as a significant similarity to the time of genetic evolutionary divergence between all populations. Thus, it seems safe to affirm that we will find similar results for the other Countries of South America, reducing speculation about the existence of rapid and silent mutations that, although there are as we have shown in this work, do not increase, until this moment, the genetic variability of the Virus, a fact that would hinder the work with molecular targets for vaccines and drugs in general.
\end{abstract}




\section{Introduction}

The new coronavirus, which originated in China, is now expanding into regions marked by poverty, lack of access to water and therefore sanitation and hygiene measures. It takes a geometric scale caused by the complete neglect of the rulers, a very common thing in almost all countries of South America. All these associated factors interfere very much in the epidemiological mechanics of the virus, accentuating the implications of the pandemic. (MILLER et al, 2020). Despite this fact, some countries in South America put in place laboratory and patient management protocols used in the SARS outbreak in 2003 and pandemic influenza in 2009, also seeking to establish communication strategies for dissemination of prophylactic measures among neighboring countries, trying to align with what is recommended and recommended by the WHO. (RODRIGUEZ-MORALES et al., 2020).

From these learned examples, we see the efficient and recent action of the Pan American Health Organization (PAHO/WHO) in the measles outbreak in Latin America, issuing constant epidemiological alerts from January 2019 to January 2020, managing to report 20,430 cases and 19 deaths in 14 countries: Argentina, Bahamas, Brazil, Chile, Colombia, Costa Rica, Cuba, Curacao, Mexico, Peru, Uruguay and Venezuela. However, despite efforts to employ protocols so far efficient in other viruses, in addition to this, the lack of effective drugs against the new coronavirus and vaccines still in the testing phase worldwide, trying to understand the evolutionary aspects of the virus, emerges as another strategy that can help the scientific community discover significant biological aspects of the virus, generating information that can be used, including, in the construction of drugs and vaccines in progress and even in the most varied prophylaxis measures (PAHO, 2020).

Thinking so, the team of the Laboratory of Population Genetics and Computational Evolutionary Biology (LaBECom-UNIVISA) conducted a study of phylogeny and molecular variance analysis to evaluate the possible levels of genetic diversity and polymorphisms existing in a PopSet of the complete genome of SARS-CoV2 from all over South America, available at the National Center for Biotechnology Information (NCBI), in the Severe acute respiratory syndrome coronavirus 2 data hub (GENBANK , 2020). 


\section{Objective}

Evaluate the possible levels of genetic diversity and polymorphism existing in 38 SARS-CoV-2 genomes in South America.

\section{Methodology}

3.1. Databank: The 38 complete genome sequences of SARS-CoV-2 from South America (Brazil, Chile, Peru, Colombia, Uruguay and Venezuela with 16, 11, 1, 2, 1, 7 haplotypes, respectively) all with 29,906 pb extension and Phred values $\geq 40$ and which now make up our study PopSet, were recovered from GENBANK (https://www.ncbi.nlm.nih.gov/labs/virus/vssi/\#/virus?SeqType_s=Nucleotide\&VirusLi neage_ss=SARS-CoV-

2,\%20taxid:2697049\&Completeness_s=complete\&Region_s=South\%20America) on August 21, 2020).

3.2. Phylogenetic analyses: Nucleotide sequences previously described were used for phylogenetic analyses. The sequences were aligned using the MEGA X program (TAMURA et al., 2018) and the gaps were extracted for the construction of phylogenetic trees.

3.3. Genetic Structuring Analyses: Paired FST estimators, Molecular Variance (AMOVA), Genetic Distance, mismatch, demographic and spatial expansion analyses, molecular diversity and evolutionary divergence time were obtained with the Software Arlequin v. 3.5 (EXCOFFIER et al., 2005) using 1000 random permutations (NEI and KUMAR, 2000). The F $\mathrm{F}_{\mathrm{ST}}$ and geographic distance matrices were not compared.

\section{Results}

\subsection{General properties of complete SARS-CoV-2 genome sequences from South} America

Of the 38 sequences of the complete genome of SARS-CoV-2 in South America with 29,996 bp extension, the analyses revealed the presence of 75 polymorphic sites and of these, only 71 sites were parsimonious-informative. The graphic representation of these sites could be seen in a logo built with the software WEBLOGO 3. (CROOKS et 
al., 2004), where the size of each nucleotide is proportional to its frequency for certain sites. (Figure 1).

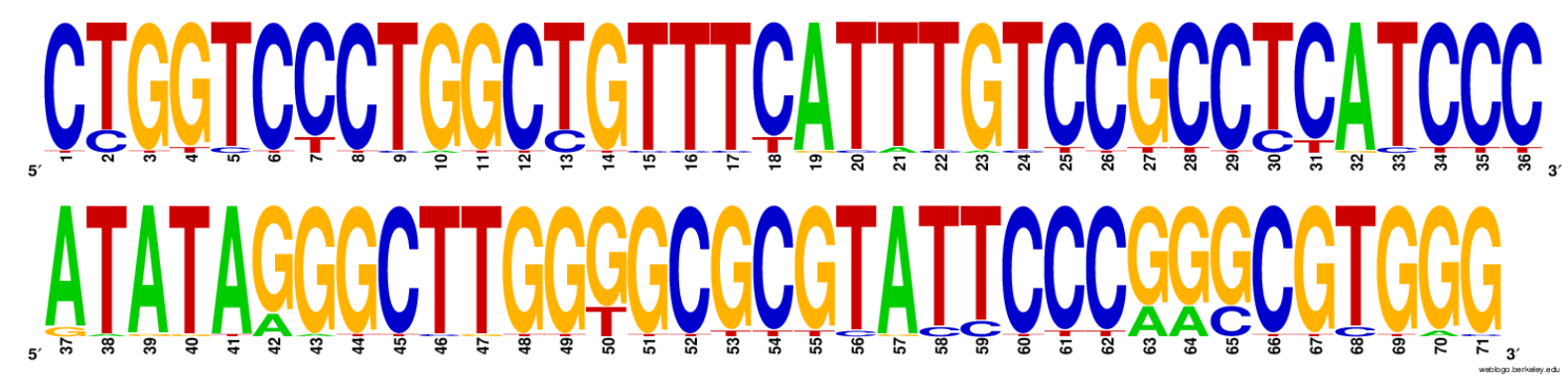

Figure 1: Graphic representation of 71 parsimonium-informative sites of complete Genome Sequences of SARS-CoV-2 from South America.

Using the UPGMA method, based on the 71 parsimony-informative sites, it was also possible to understand that the 38 haplotypes comprised very similar groups genetically and with a non-significant polymorphism pattern (Figure 2). 


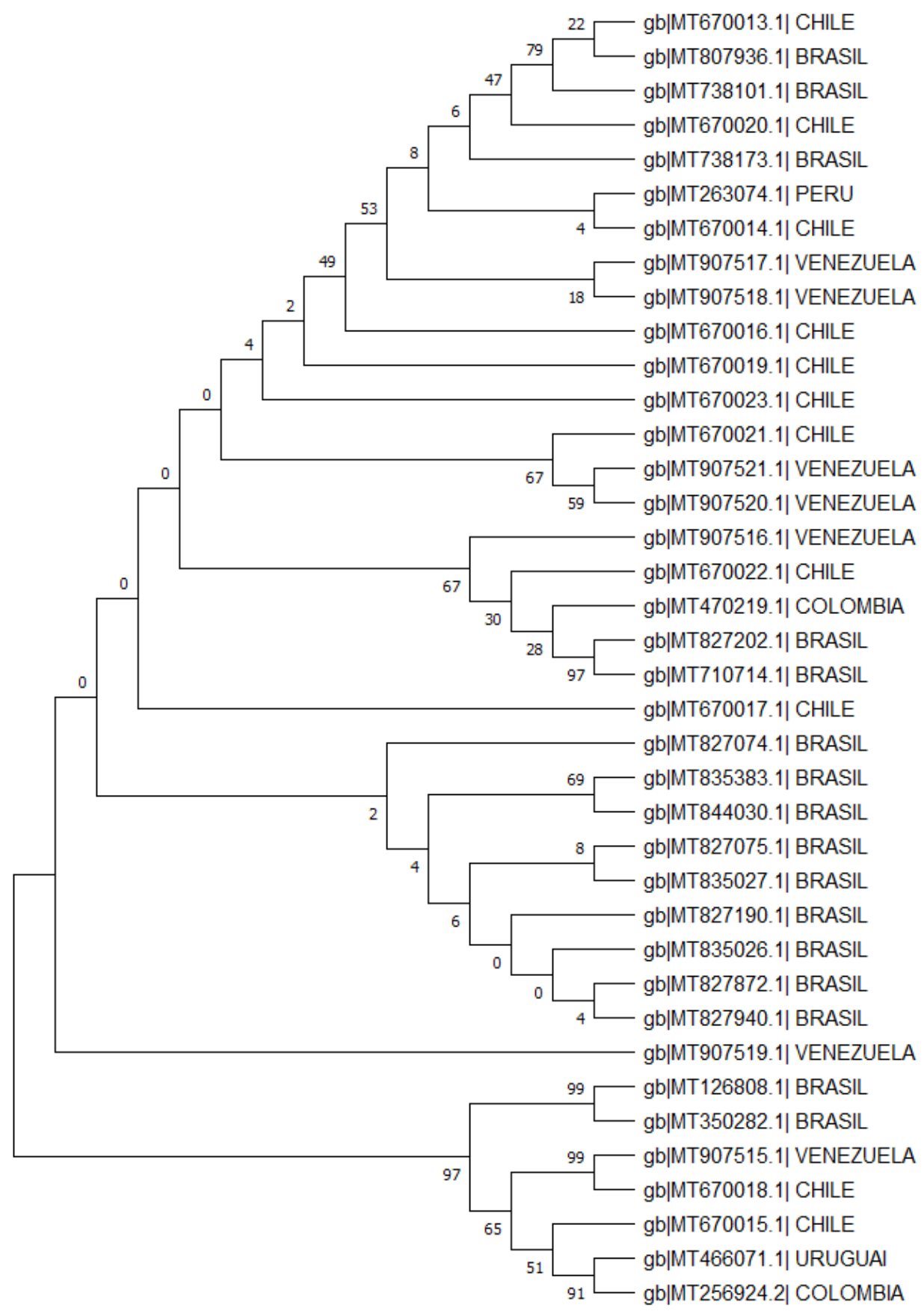

Figure 2. Evolutionary analysis by the maximum likelihood method. The evolutionary history was inferred using the maximum likelihood method and the 3-parameter Tamura model [1]. The tree with the highest probability of logging (-1366.35) is shown. The percentage of trees in which the associated dollar sums group together is shown next to the branches. The initial trees for heuristic research were obtained automatically by applying the Join-Join and BioNJ algorithms to an array of distances in estimated pairs using the Tamura 3 parameter model and then selecting the topology with a higher $\log$ probability value. This analysis involved 38 nucleotide sequences. The evolutionary analyses were performed in MEGA X 


\subsection{Molecular Variance Analysis (AMOVA) and Genetic Distance}

Genetic distance and molecular variation (AMOVA) analyses were not significant for the groups studied, presenting a variation component of 0.12 between populations and 4.46 within populations. The $\mathrm{F}_{\mathrm{ST}}$ value $(0.03)$ showed a low fixation index, with nonsignificant evolutionary divergences within and between groups (Table 1 and Figure 3 ).

A significant similarity was also evidenced for the time of genetic evolutionary divergence among all populations; supported by $\tau$ variations, mismatch analyses and demographic and spatial expansion analyses. With a representative exception for haplotypes from Brazil and Venezuela (Table 2), (Figures 4 and 5).

Table 1. Components of haplotypic variation and paired $\mathrm{F}_{\mathrm{ST}}$ value for the 38 complete genome sequences of SARS-CoV-2 from South America.

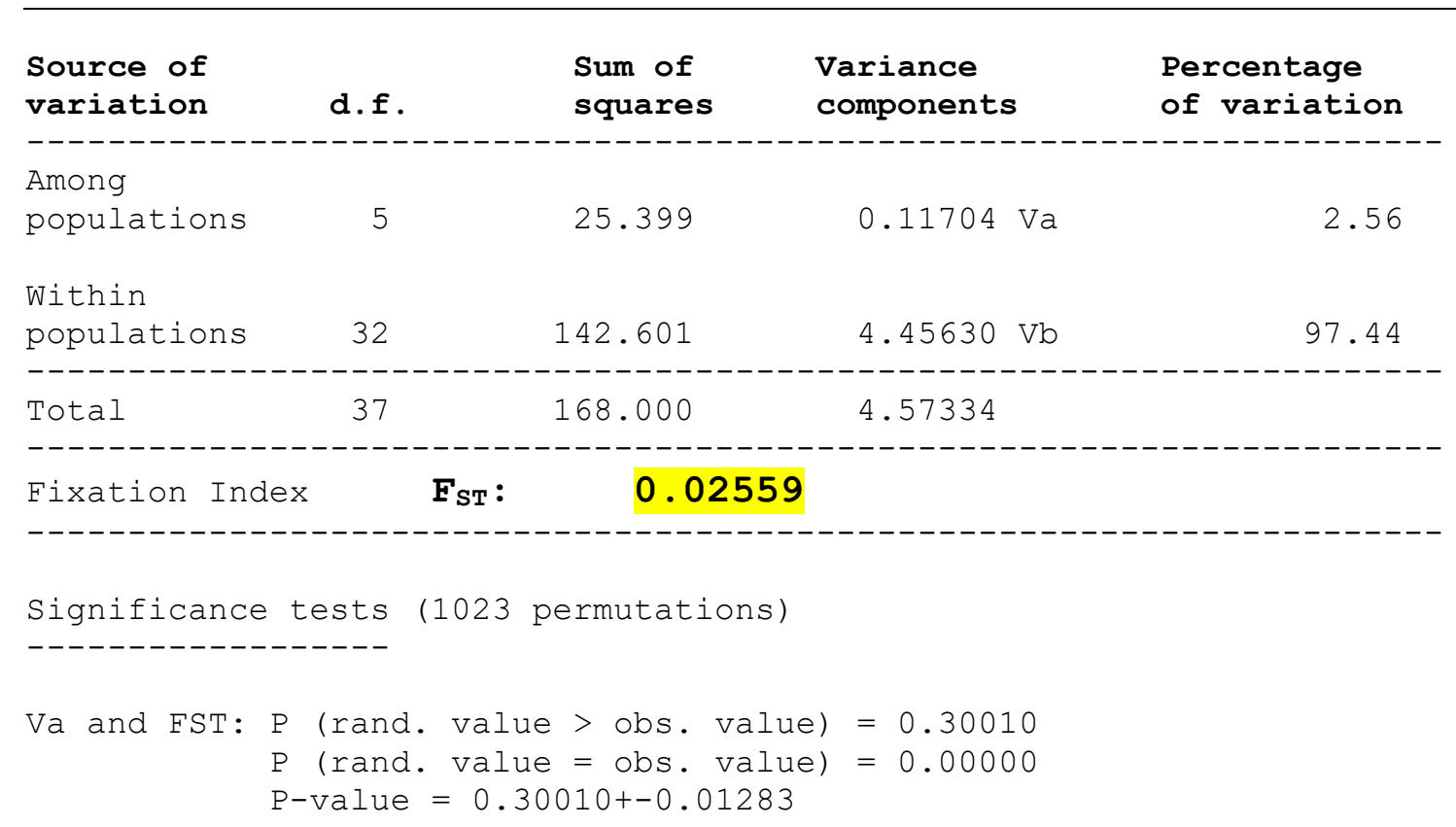




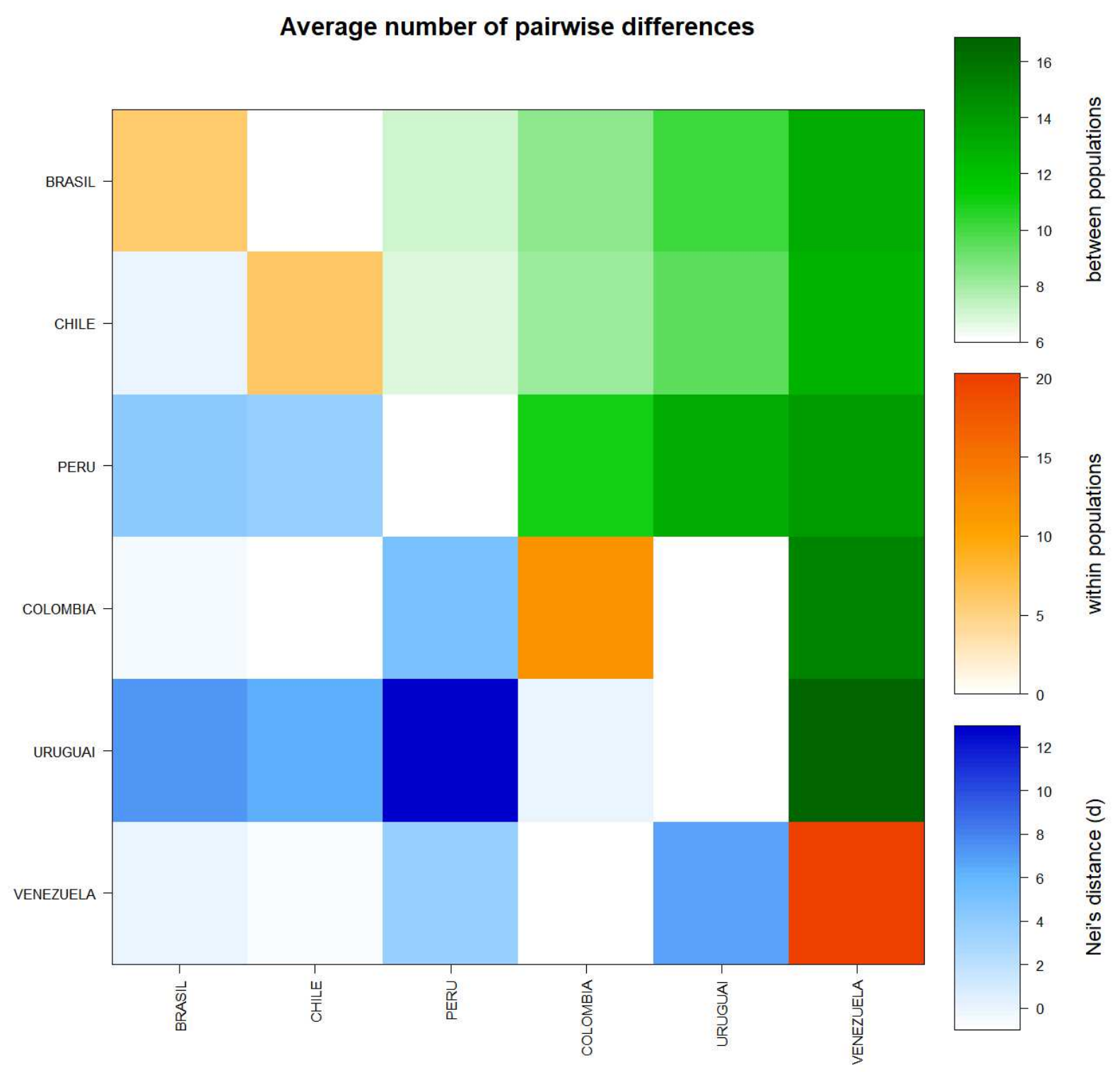

Figure 3. Matrix of paired differences between the populations studied: between the groups; within the groups; and Nei distance for the complete genome sequences of SARS-CoV-2 from six countries in South America. 
Table 2. Demographic and spatial expansion simulations based on the $\tau, \theta$, and $\mathrm{M}$ indices of sequences of the complete SARS-CoV-2 genomes from six South American countries.

\begin{tabular}{|c|c|c|c|c|c|c|c|c|}
\hline Statistics & BRASIL & CHILE & PERU & COLOMBIA & URUGUAI & VENEZUELA & Mean & s.d. \\
\hline \multicolumn{9}{|l|}{ Demographic expansion } \\
\hline Tau & 8.65821 & 3.41406 & 0.00000 & 0.00000 & 0.00000 & 8.00000 & 3.34538 & 4.08585 \\
\hline Tau qt $2.5 \%$ & 1.43937 & 0.00000 & 0.00000 & 0.00000 & 0.00000 & 4.43744 & 0.97947 & 1.78922 \\
\hline Tau qt $5 \%$ & 2.76561 & 2.33788 & 0.00000 & 0.00000 & 0.00000 & 5.36523 & 1.74479 & 2.17413 \\
\hline Tau qt 95\% & 12.31057 & 17.19734 & 0.00000 & 0.00000 & 0.00000 & 20.92787 & 8.40596 & 9.60534 \\
\hline Tau qt $97.5 \%$ & 13.72265 & 18.72268 & 0.00000 & 0.00000 & 0.00000 & 21.95513 & 9.06674 & 10.27271 \\
\hline Theta0 qt $2.5 \%$ & 0.00000 & 0.00000 & 0.00000 & 0.00000 & 0.00000 & 0.00000 & 0.00000 & 0.00000 \\
\hline Theta 0 qt $5 \%$ & 0.00000 & 0.00000 & 0.00000 & 0.00000 & 0.00000 & 0.00000 & 0.00000 & 0.00000 \\
\hline Theta0 qt 95\% & 1.82648 & 3.26617 & 0.00000 & 0.00000 & 0.00000 & 12.13044 & 2.87052 & 4.72678 \\
\hline Theta 0 qt $97.5 \%$ & 2.83189 & 4.79179 & 0.00000 & 0.00000 & 0.00000 & 16.98018 & 4.10064 & 6.60932 \\
\hline Thetal & 8.67921 & 13.59864 & 0.00000 & 0.00000 & 0.00000 & 3414.97837 & 572.87604 & 1392.35166 \\
\hline Thetal qt $2.5 \%$ & 3.11981 & 3.91268 & 0.00000 & 0.00000 & 0.00000 & 27.98066 & 5.83553 & 10.98763 \\
\hline Thetal qt $5 \%$ & 4.26270 & 4.56568 & 0.00000 & 0.00000 & 0.00000 & 39.51658 & 8.05749 & 15.56301 \\
\hline Thetal qt 95\% & 33.99159 & 82.50502 & 0.00000 & 0.00000 & 0.00000 & 323.11280 & 73.26823 & 126.61357 \\
\hline Thetal qt $97.5 \%$ & 52.81974 & 162.02985 & 0.00000 & 0.00000 & 0.00000 & 590.61070 & 134.24338 & 232.26574 \\
\hline Raggedness index & 0.07035 & 0.00860 & 0.00000 & 0.00000 & 0.00000 & 0.16780 & 0.04112 & 0.06787 \\
\hline Raggedness p-value & 0.38000 & 1.00000 & 0.00000 & 0.00000 & 0.00000 & 0.22000 & 0.26667 & 0.39144 \\
\hline \multicolumn{9}{|l|}{ Spatial expansion } \\
\hline Tau & 6.18844 & 2.25056 & 0.00000 & 0.00000 & 0.00000 & 8.24067 & 2.77994 & 3.60283 \\
\hline Tau qt $2.5 \%$ & 1.28581 & 0.69166 & 0.00000 & 0.00000 & 0.00000 & 3.44275 & 0.90337 & 1.34817 \\
\hline Tau qt 5\% & 3.98499 & 1.97354 & 0.00000 & 0.00000 & 0.00000 & 4.37483 & 1.72223 & 2.05513 \\
\hline Tau qt 95\% & 10.32285 & 13.44850 & 0.00000 & 0.00000 & 0.00000 & 14.53488 & 6.38437 & 7.12916 \\
\hline Tau qt $97.5 \%$ & 10.82249 & 17.56023 & 0.00000 & 0.00000 & 0.00000 & 15.32114 & 7.28398 & 8.26907 \\
\hline Theta & 1.64652 & 4.96606 & 0.00000 & 0.00000 & 0.00000 & 5.15534 & 1.96132 & 2.48474 \\
\hline Theta qt $2.5 \%$ & 0.00072 & 0.00072 & 0.00000 & 0.00000 & 0.00000 & 0.00072 & 0.00036 & 0.00040 \\
\hline Theta qt $5 \%$ & 0.00072 & 0.00072 & 0.00000 & 0.00000 & 0.00000 & 0.00258 & 0.00067 & 0.00100 \\
\hline Theta qt 95\% & 2.75007 & 7.34736 & 0.00000 & 0.00000 & 0.00000 & 16.21983 & 4.38621 & 6.46833 \\
\hline
\end{tabular}


Theta qt $97.5 \%$

$$
\begin{array}{r}
\text { M } \\
\text { M qt 2.5\% } \\
\text { M qt 5\% } \\
\text { M qt } 95 \% \\
\text { M qt } 97.5 \% \\
\text { SSD }
\end{array}
$$

Model (SSD) p-value

Raggedness index

Raggedness p-value
3.02024

2.30868

0.52435

0.82693

12.86742

15.34689

0.02288

0.77000

0.07035

0.68000

11.22560

0.86722

1.18551

110.88566

191.55404

0.00560

0.98000

0.00860

1.00000
0.00000

0.00000

0.00000

0.00000

0.00000

0.00000

0.00000

0.00000

0.00000

0.00000
0.00000

0.00000

0.00000

0.00000

0.00000

0.00000

0.00000

0.00000

0.00000

0.00000
0.00000

0.00000

0.00000

0.00000

0.00000

0.00000

0.00000

0.00000

0.00000

0.00000
20.05757

8827.30237

20.59232

33.82173

2097.48882

5327.87210

0.07137

0.10000

0.16780

0.22000
5.12126 473.47278

3.66398

5.97236

370.20698

2159.51526

$0.01664 \quad 0.02824$

$0.30833 \quad 0.44562$

$0.04112 \quad 0.06787$

$0.31667 \quad 0.42641$ 

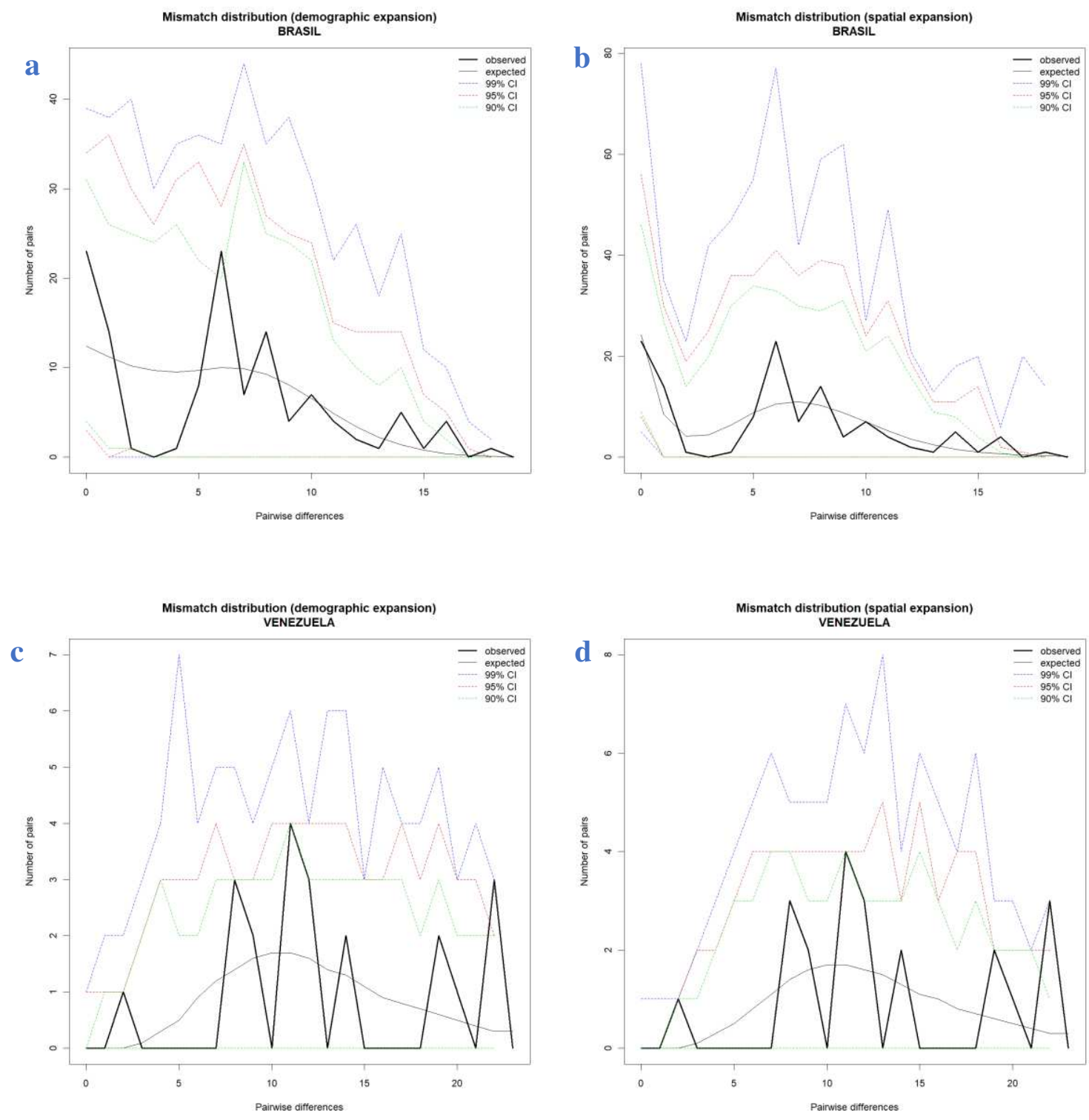

Figure 4. Comparison between the Demographic and Spatial Expansion of sequences of the complete genomes of SARS-CoV-2 from six countries in South America. (a and b) Graphs of demographic expansion and spatial expansion of haplotypes from Brazil, respectively; (c and d) Graphs of demographic expansion and spatial expansion of haplotypes from Venezuela, respectively. *Graphs Generated by the statistical package in R language using the output data of the Software Arlequin version 3.5.1.2 


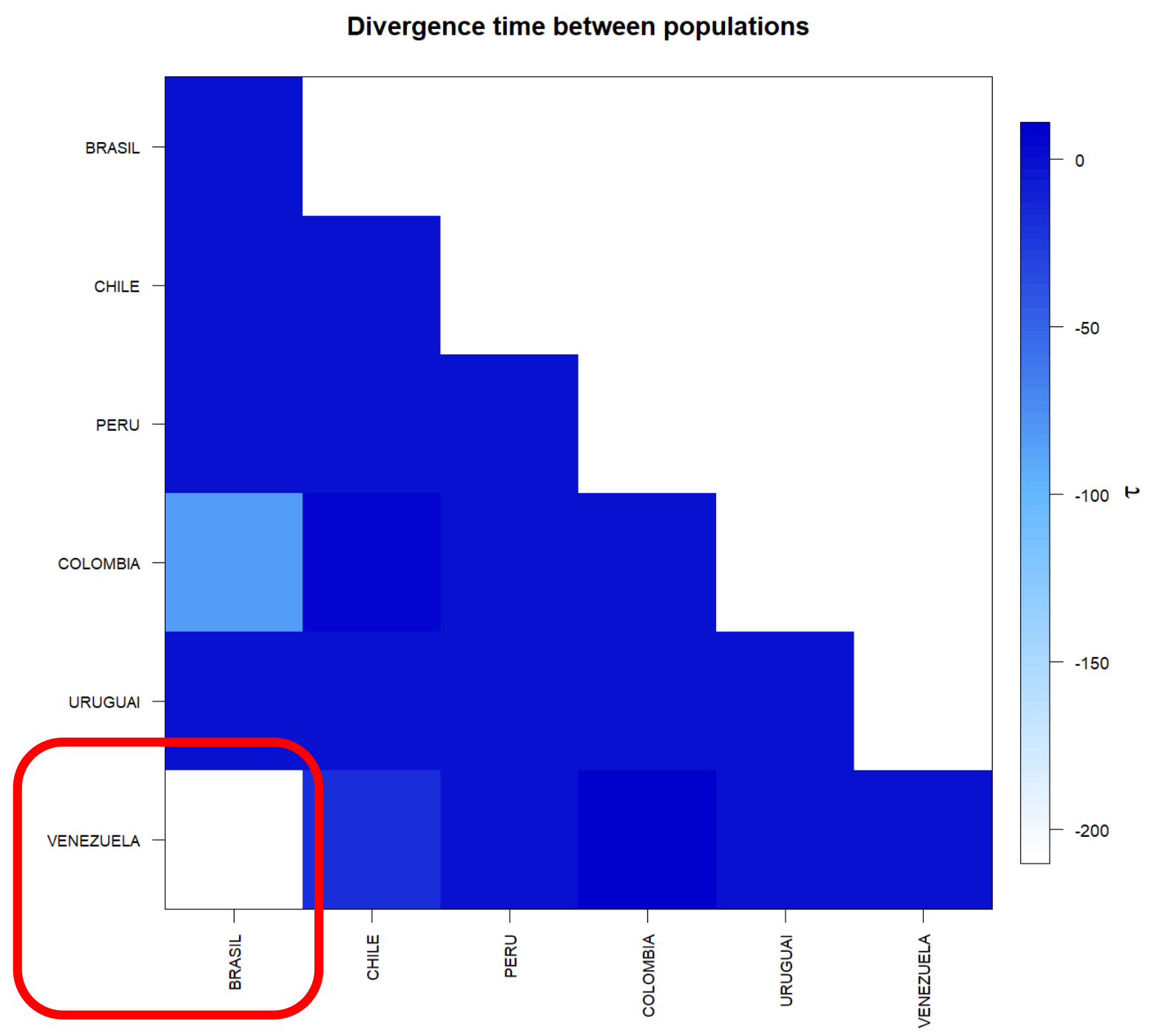

Figure 5. Matrix of divergence time between the complete genomes of SARS-CoV-2 from six countries in South America. In evidence the high value $\tau$ present between the sequences of Brazil and Venezuela. * Generated by the statistical package in $\mathrm{R}$ language using the output data of the Software Arlequin version 3.5.1.2.

The molecular diversity analyses estimated per $\theta$ reflected a significant level of mutations among all haplotypes (transitions and transversions). Indel mutations (insertions or additions) were not found in any of the six groups studied. The D tests of Tajima and Fs de Fu showed disagreements between the estimates of general $\theta$ and $\pi$, but with negative and highly significant values, indicating, once again, an absence of population expansions. The irregularity index ( $R=$ Raggedness $)$ with parametric bootstrap, simulated new $\theta$ values for before and after a supposed demographic expansion and in this case assumed a value equal to zero for all groups (Table 6); (Figure 6). 
Table 3. Molecular Diversity Indices for the complete Genomes of SARS-CoV-2 from six countries in South America

\begin{tabular}{|c|c|c|c|c|c|c|c|c|}
\hline Statistics & BRASIL & CHILE & PERU & COLOMBIA & URUGUAI & VENEZUELA & Mean & s.d. \\
\hline No. of transitions & 21 & 16 & 0 & 9 & 0 & 28 & 12.333 & 11.396 \\
\hline No. of transversions & 7 & 2 & 0 & 3 & 0 & 14 & 4.333 & 5.391 \\
\hline No. of substitutions & 28 & 18 & 0 & 12 & 0 & 42 & 16.667 & 16.428 \\
\hline No. of indels & 0 & 0 & 0 & 0 & 0 & 0 & 0.000 & 0.000 \\
\hline No. of ts. sites & 21 & 16 & 0 & 9 & 0 & 28 & 12.333 & 11.396 \\
\hline No. of tv. sites & 7 & 2 & 0 & 3 & 0 & 14 & 4.333 & 5.391 \\
\hline No. of subst. sites & 28 & 18 & 0 & 12 & 0 & 42 & 16.667 & 16.428 \\
\hline No. private subst. sites & 20 & 5 & 0 & 4 & 0 & 27 & 9.333 & 11.378 \\
\hline No. of indel sites & 0 & 0 & 0 & 0 & 0 & 0 & 0.000 & 0.000 \\
\hline Pi & 5.942 & 6.236 & 0.000 & 12.000 & 0.000 & 13.143 & 6.22015 & 5.63540 \\
\hline Theta_k & N.A. & N.A. & N.A. & N.A. & N.A. & N.A. & N.A. & N.A. \\
\hline Theta_k_lower & N.A. & N.A. & N.A. & N.A. & N.A. & N.A. & N.A. & N.A. \\
\hline Theta_k_upper & N.A. & N.A. & N.A. & N.A. & N.A. & N.A. & N.A. & N.A. \\
\hline Theta_H & N.A. & N.A. & N.A. & N.A. & N.A. & N.A. & N.A. & N.A. \\
\hline s.d. Theta_H & N.A. & N.A. & N.A. & N.A. & N.A. & N.A. & N.A. & N.A. \\
\hline Theta_S & 8.43824 & 6.14551 & 0.00000 & 12.00000 & 0.00000 & 17.14286 & 7.28777 & 6.75543 \\
\hline s.d. Theta_S & 3.34086 & 2.74879 & 0.00000 & 8.83176 & 0.00000 & 8.00564 & 3.82118 & 3.82620 \\
\hline Theta_pi & 5.94167 & 6.23636 & 0.00000 & 12.00000 & 0.00000 & 13.14286 & 6.22015 & 5.63540 \\
\hline s.d. Theta_pi & 3.35170 & 3.61960 & 0.00000 & 12.49000 & 0.00000 & 7.73070 & 4.53200 & 4.83456 \\
\hline
\end{tabular}




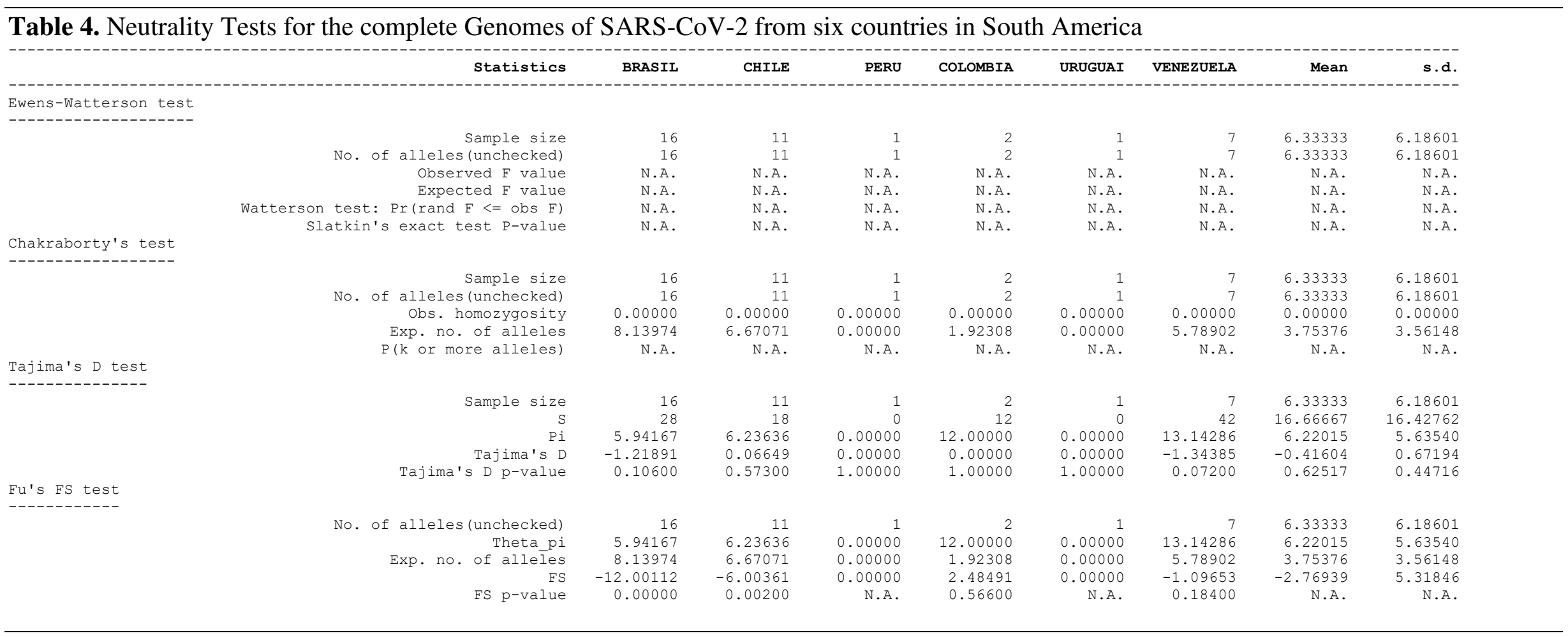




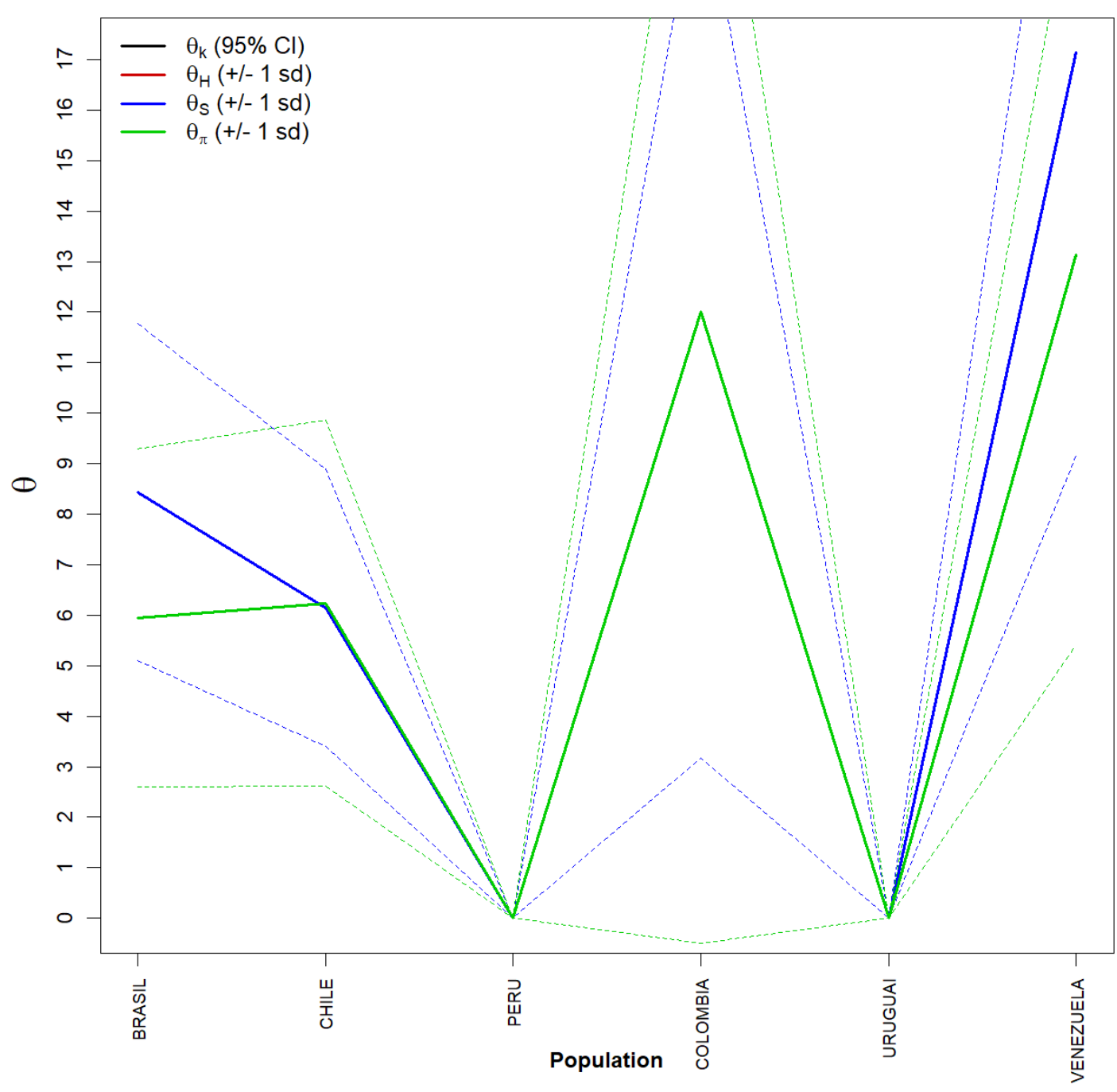

Figure 6. Graph of molecular diversity indices for the complete genomes of SARS-CoV-2 from six countries in South America. In the graph the values of $\theta:(\theta \mathrm{k})$ Relationship between the expected number of alllos $(\mathrm{k})$ and the sample size; $(\theta \mathrm{H})$ Expected homozygosity in a balanced relationship between drift and mutation; $(\theta \mathrm{S})$ Relationship between the number of segregating sites $(\mathrm{S})$, sample size (n) and nonrecombinant sites; $(\theta \pi)$ Relationship between the average number of paired differences $(\pi)$ and $\theta$. * Generated by the statistical package in $\mathrm{R}$ language using the output data of the Arlequin software version 3.5.1.2.

\section{Discussion}

As the use of phylogenetic analysis and population structure methodologies had not yet been used in this PopSet, in this study it was possible to detect the existence of 6 distinct groups for the complete genome sequences of SARS-CoV-2 from South America, but with minimal variations among all of them. The groups described here presented 
minimum structuring patterns and were effectively slightly higher for the populations of Brazil and Venezuela. These data suggest that the relative degree of structuring present in these two countries may be related to gene flow. These structuring levels were also supported by simple phylogenetic pairing methodologies such as UPGMA, which in this case, with a discontinuous pattern of genetic divergence between the groups (supports the idea of possible sub-geographical isolations resulting from past fragmentation events), was observed a not so numerous amount of branches in the tree generated and with few mutational steps.

These few mutations have possibly not yet been fixed by drift by the lack of the founding effect, which accompanies the behavior of dispersion and/or loss of intermediate haplotypes throughout the generations. The values found for genetic distance support the presence of this continuous pattern of low divergence between the groups studied, since they considered important the minimum differences between the groups, when the haplotypes between them were exchanged, as well as the inference of values greater than or equal to that observed in the proportion of these permutations, including the p-value of the test.

The discrimination of the 38 genetic entities in their localities was also perceived by their small inter-haplotypic variations, hierarchised in all covariance components: by their intra- and inter-individual differences or by their intra- and intergroup differences, generating a dendogram that supports the idea that the significant differences found in countries such as Brazil and Venezuela, for example, were shared more in their form than in their number, since the result of estimates of the average evolutionary divergence found within these and other countries, even if they exist, were very low.

Based on the high level of haplotypic sharing, tests that measure the relationship between genetic distance and geographic distance, such as the Mantel test, were dispensed in this Estimators $\theta$, even though they are extremely sensitive to any form of molecular variation (FU, 1997), supported the uniformity between the results found by all the methodologies employed, and can be interpreted as a phylogenetic confirmation that there is a consensus in the conservation of the SARS-CoV-2 genome in the Countries of America of America of South objects of this study, being therefore safe to affirm that the small number of existing polymorphisms should be reflected even in all their protein products. This consideration provides the safety that, although there are differences in the haplotypes studied, these differences are minimal in geographically distinct regions and 
thus it seems safe to extrapolate the levels of polymorphism and molecular diversity found in the samples of this study to other genomes of other South American countries, reducing speculation about the existence of rapid and silent mutations that, although they exist as we have shown in this work, they can significantly increase the genetic variability of the Virus, making it difficult to work with molecular targets for vaccines and drugs in general.

\section{References}

Crooks G.E., Hon G., Chandonia J.M., Brenner S.E. WebLogo: A sequence logo generator, Genome Research, 14:1188-1190, (2004).

Fu, Y.X. Statistical tests of neutrality of mutations against population growth, hitchhiking and background selection. Genetics 147: 915-925. (1997)

GenBank [Internet]. Bethesda (MD): National Library of Medicine (US), National Center for Biotechnology Information; [1982] - [cited 2020 Aug 21]. Available from: https://www.ncbi.nlm.nih.gov/labs/virus/vssi/\#/virus?SeqType_s=Nucleotide\&VirusLin eage $\mathrm{ss}=\mathrm{SARS}-\mathrm{CoV}$ -

2,\%20taxid:2697049\&Completeness_s=complete\&Region_s=South\%20America

Kumar S, Stecher G, Li M, Knyaz C; Tamura K. MEGA X: Molecular Evolutionary Genetics Analysis across computing platforms. (2018). Molecular Biology and Evolution 35:1547-1549.

Miller MJ, Loaiza JR, Takyar A, Gilman RH. COVID-19 na América Latina: Nova dinâmica de transmissão para uma pandemia global? PLoS Negl Trop Dis. 2020;14(5):e0008265. Publicado em 7 de maio de 2020. doi:10.1371/journal.pntd.0008265

PAHO. Epidemiological update measles - 24 january 2020. https://www.paho.org/hq/index.php?option=com_docman\&view=download\&category $\underline{\text { slug=measles-2204\&alias=51389-24-january-2020-measles-epidemiological-update- }}$ $\underline{1 \& \text { Itemid=270\&lang=en } .2020}$ 
Rodriguez-Morales, A. J.; Gallego, V.; Escalera-Antezana, J. P.; Méndez, C.A.; Zambrano, L. I.; Franco-Paredes, C.; Suárez, J. A.; Rodriguez-Enciso, H. D.; , BalbinRamon, G. J.; Savio-Larriera, E.; Risquez, A.; Cimerman, S. COVID-19 in Latin America: The implications of the first confirmed case in Brazil. Travel Med Infect Dis. 2020 May-June; 35: 101613. Published online 2020 Feb 29. doi: 10.1016/j.tmaid.2020.101613 PMCID: PMC7129040

Tamura K. Estimation of the number of nucleotide substitutions when there are strong transition-transversion and $\mathbf{G}+\mathbf{C}$-content biases. Molecular Biology and Evolution 9:678-687. (1992). 


\section{STATEMENT}

Me and the other authors of the manuscript "Molecular variance analysis (AMOVA) and levels of genetic diversity of complete genome of SARS-CoV-2 virus from of six South American Countries" declare that there are no competing interests. We are all from the same laboratory and the work was carried out together.

\section{Pierre Teodosio Felix}

Head of research Laboratory of Population Genetics and Computational Evolutionary Biology LaBECom, UNIVISA, Vitória de Santo Antão, Pernambuco 
Figures

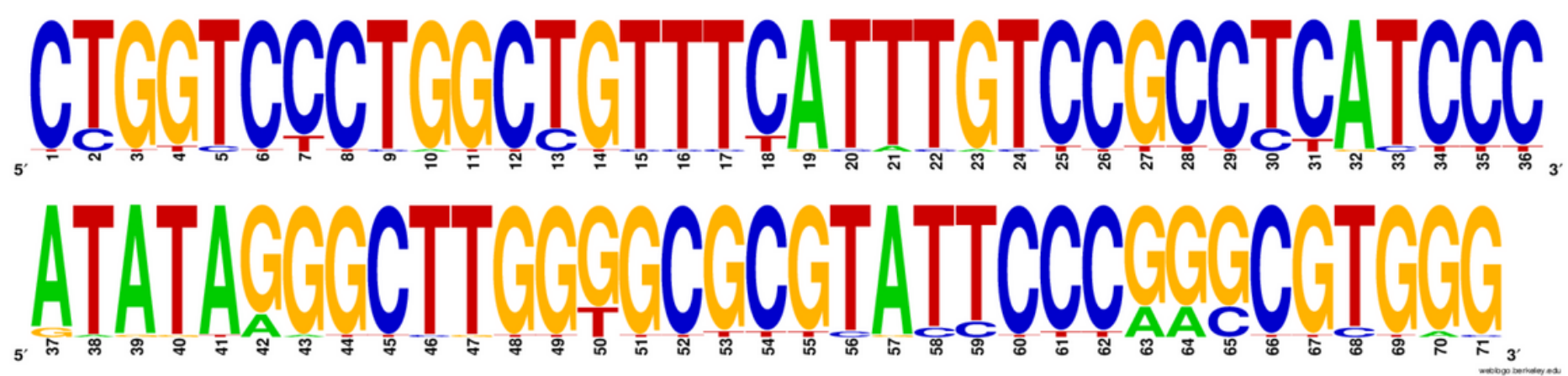

Figure 1

Graphic representation of 71 parsimonium-informative sites of complete Genome Sequences of SARSCoV-2 from South America. 


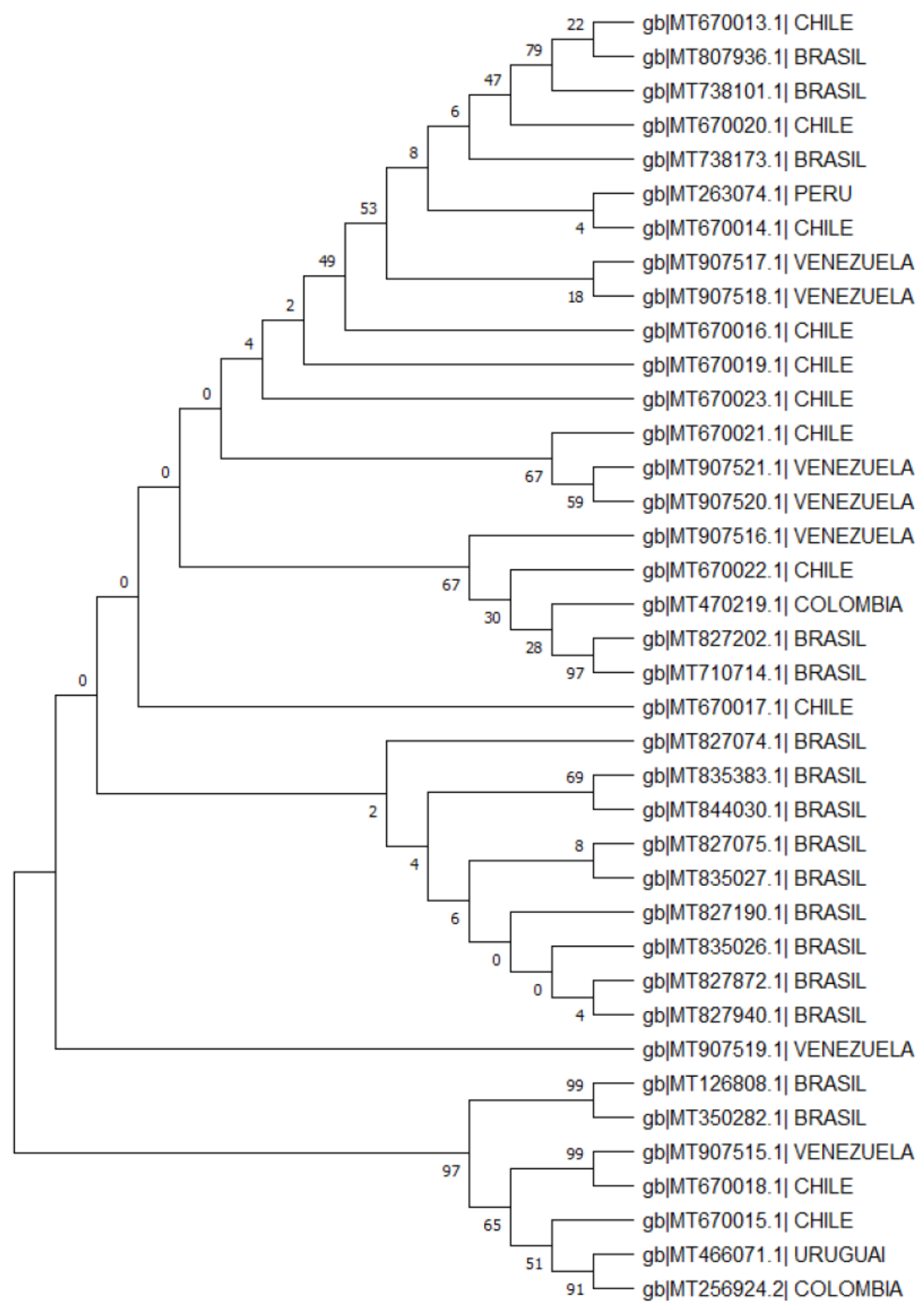

Figure 2

Evolutionary analysis by the maximum likelihood method. The evolutionary history was inferred using the maximum likelihood method and the 3-parameter Tamura model [1]. The tree with the highest probability of logging (-1366.35) is shown. The percentage of trees in which the associated dollar sums group together is shown next to the branches. The initial trees for heuristic research were obtained automatically by applying the Join-Join and BioNJ algorithms to an array of distances in estimated pairs 
using the Tamura 3 parameter model and then selecting the topology with a higher log probability value. This analysis involved 38 nucleotide sequences. The evolutionary analyses were performed in MEGA X

\section{Average number of pairwise differences}

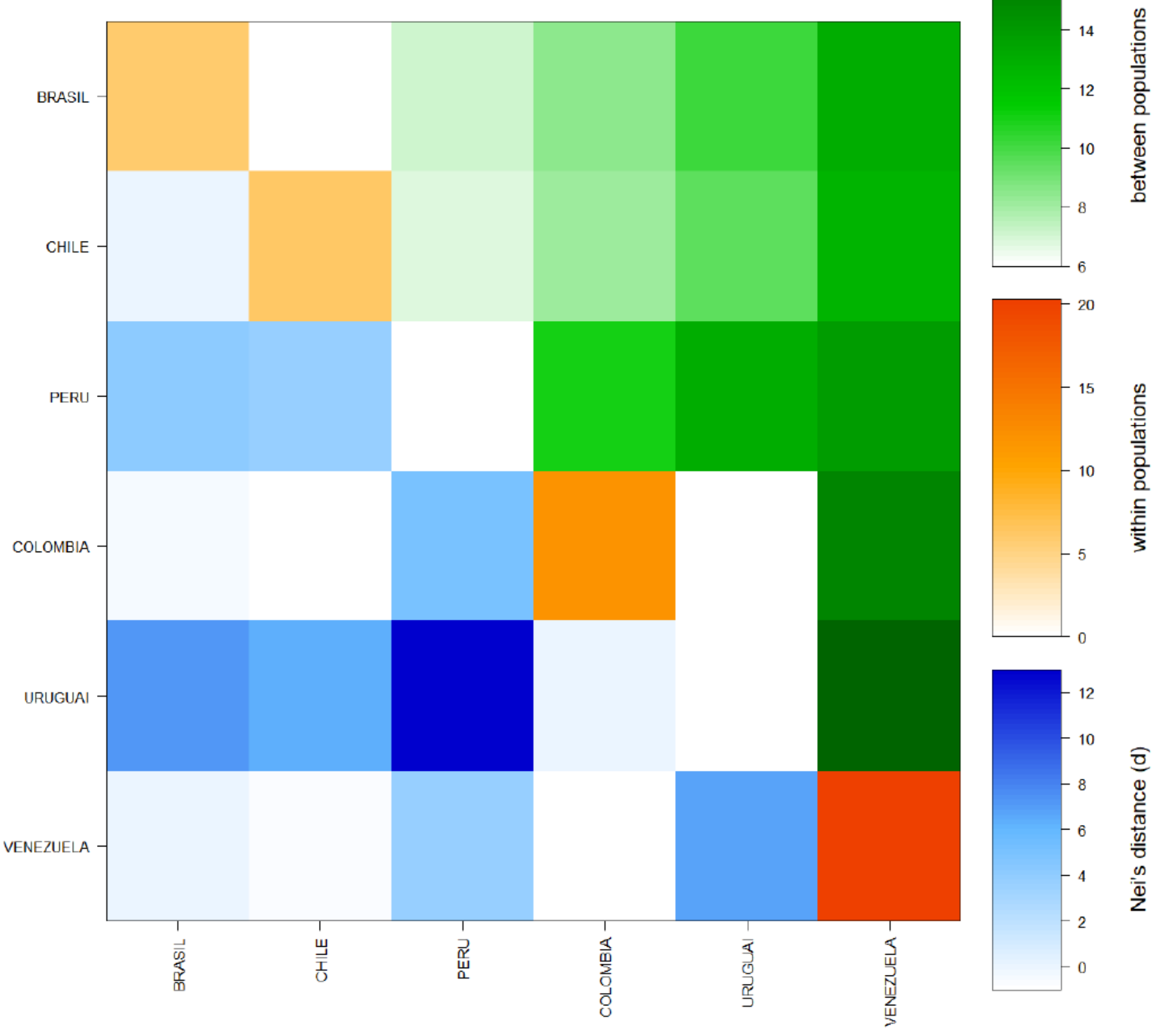

\section{Figure 3}

Matrix of paired differences between the populations studied: between the groups; within the groups; and Nei distance for the complete genome sequences of SARS-CoV-2 from six countries in South America. 

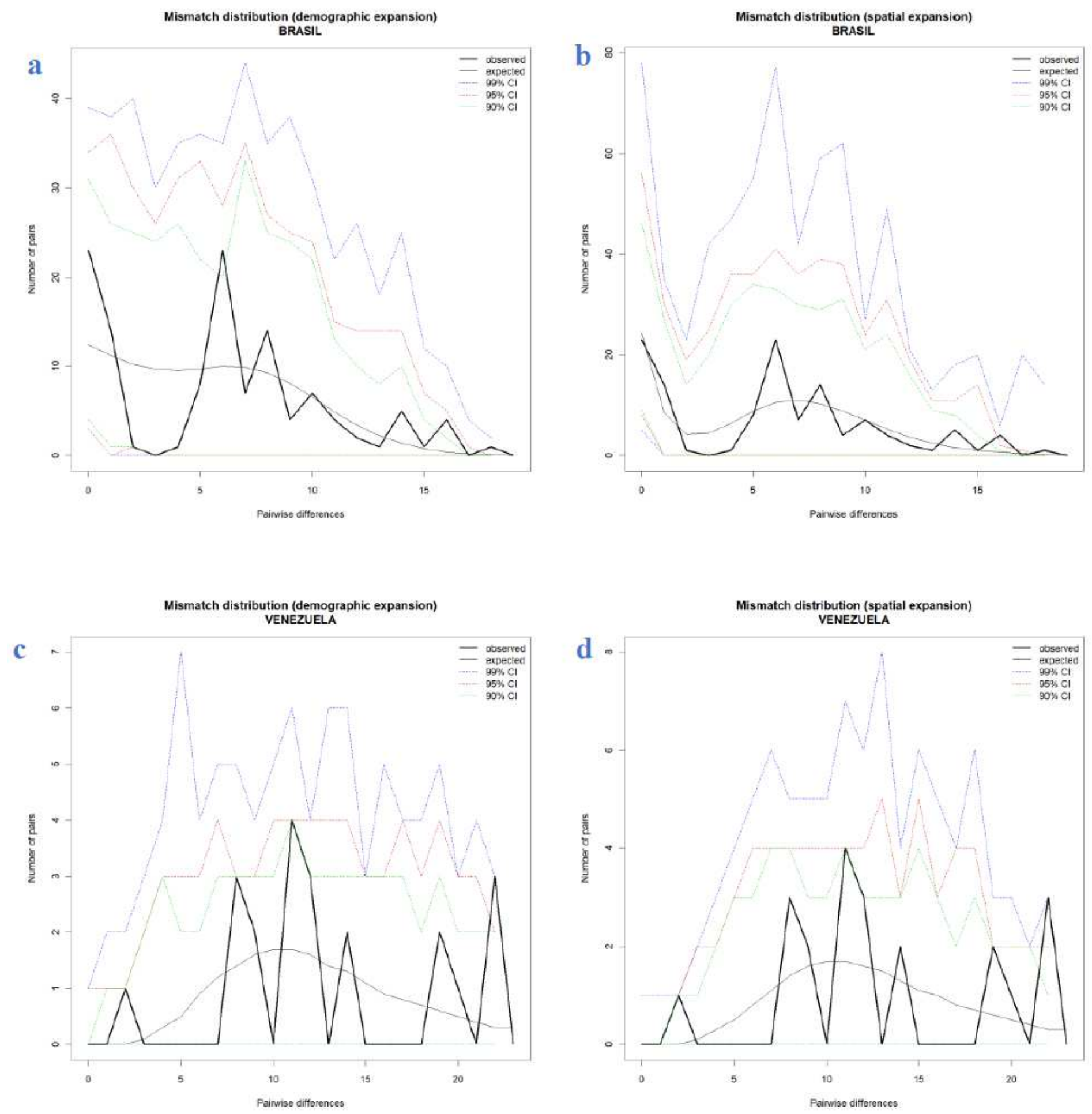

Figure 4

Comparison between the Demographic and Spatial Expansion of sequences of the complete genomes of SARS-CoV-2 from six countries in South America. ( $a$ and b) Graphs of demographic expansion and spatial expansion of haplotypes from Brazil, respectively; (c and d) Graphs of demographic expansion and spatial expansion of haplotypes from Venezuela, respectively. ${ }^{*}$ Graphs Generated by the statistical package in $\mathrm{R}$ language using the output data of the Software Arlequin version 3.5.1.2 
Divergence time between populations

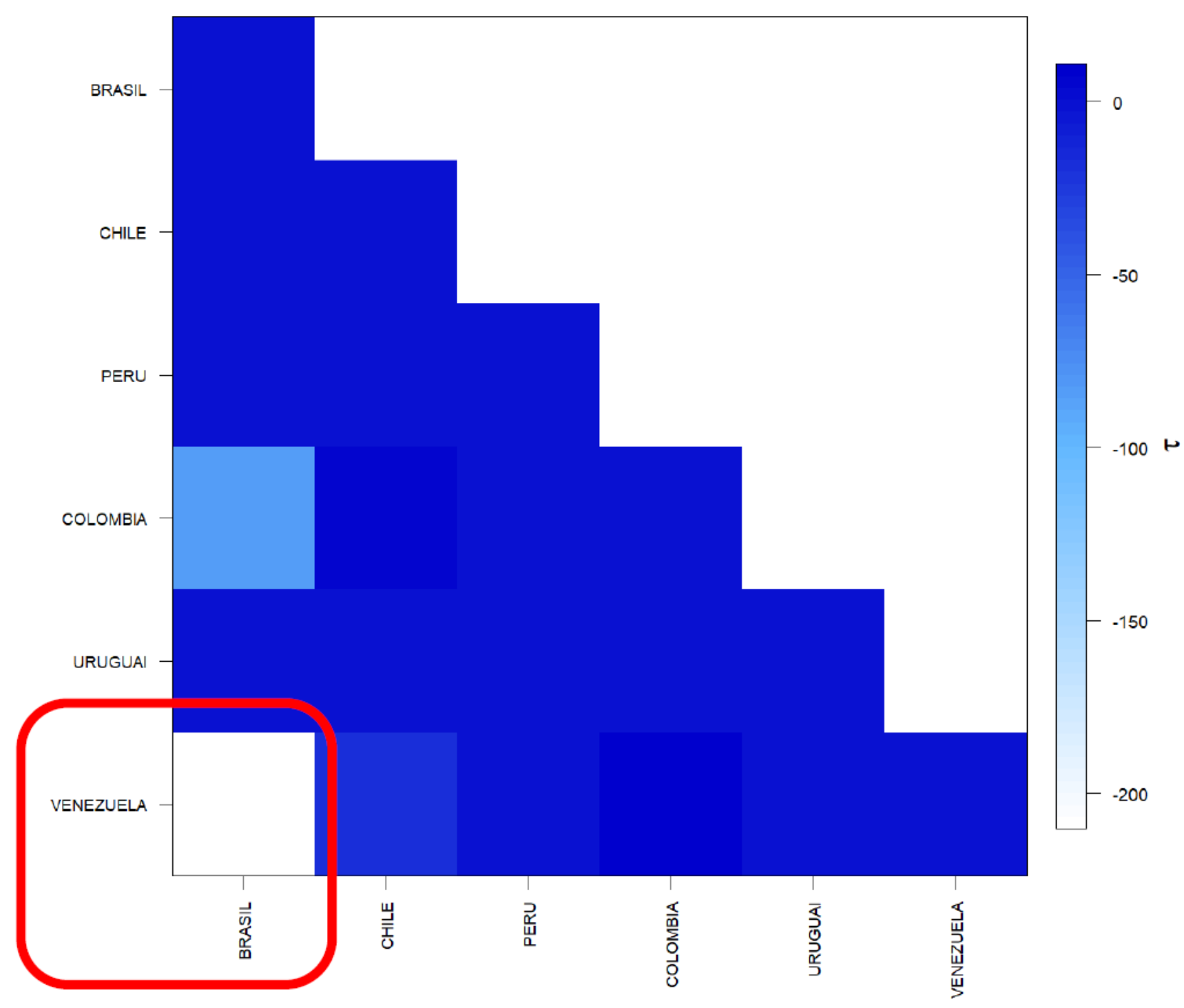

\section{Figure 5}

Matrix of divergence time between the complete genomes of SARS-CoV-2 from six countries in South America. In evidence the high value $\tau$ present between the sequences of Brazil and Venezuela. * Generated by the statistical package in $\mathrm{R}$ language using the output data of the Software Arlequin version 3.5.1.2. 


\section{Molecular diversity indices}

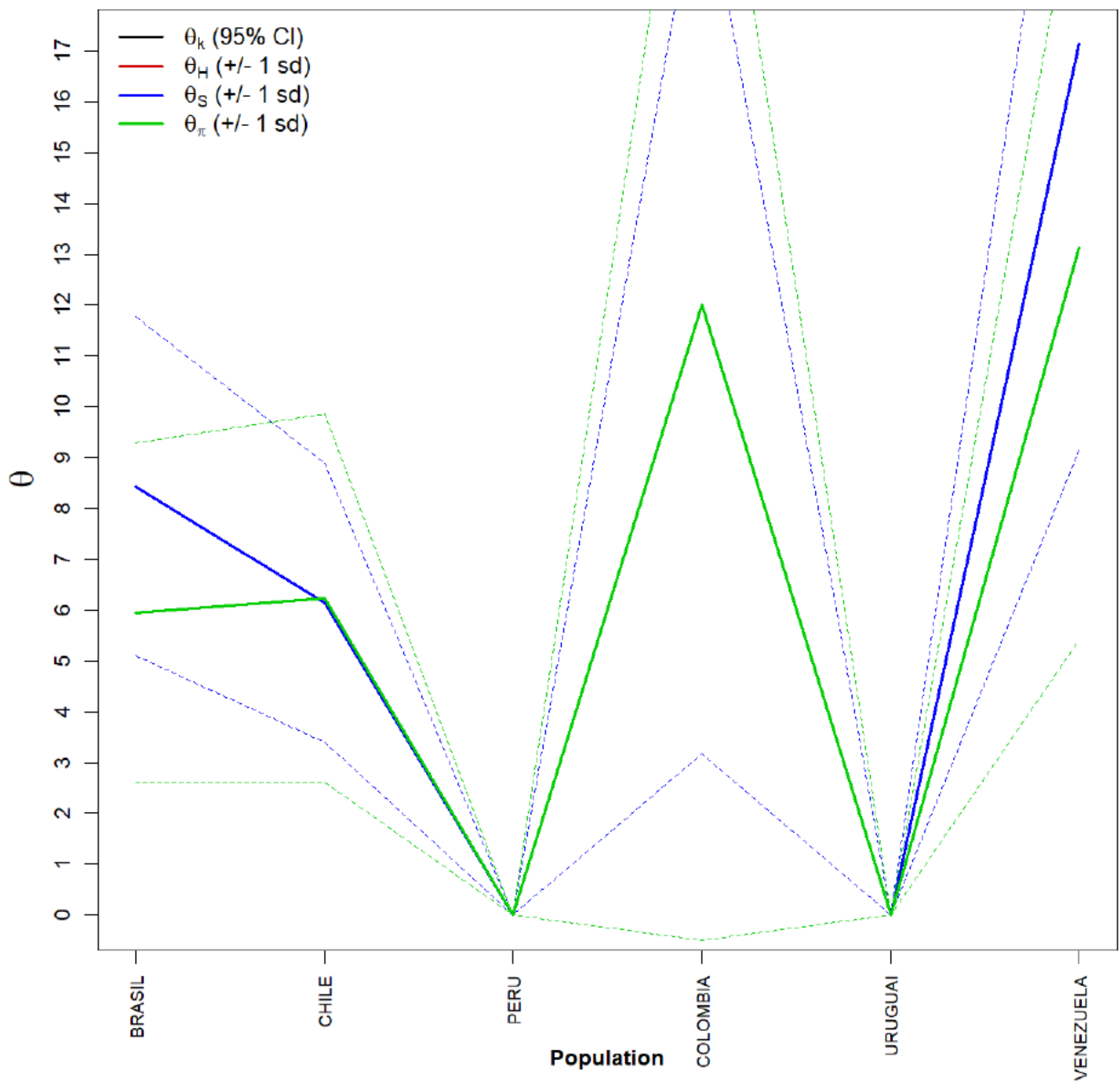

Figure 6

Graph of molecular diversity indices for the complete genomes of SARS-CoV-2 from six countries in South America. In the graph the values of $\theta$ : $(\theta \mathrm{k})$ Relationship between the expected number of alllos $(\mathrm{k})$ and the sample size; $(\theta \mathrm{H})$ Expected homozygosity in a balanced relationship between drift and mutation; $(\theta S)$ Relationship between the number of segregating sites $(S)$, sample size $(n)$ and non-recombinant sites; $(\theta \pi)$ Relationship between the average number of paired differences $(\pi)$ and $\theta .{ }^{*}$ Generated by the statistical package in $\mathrm{R}$ language using the output data of the Arlequin software version 3.5.1.2. 